\title{
The least core in fixed-income taxation models: a brief mathematical inspection
}

\author{
Paula Curt ${ }^{1}$, Cristian M Litan ${ }^{1}$ and Diana Andrada Filip ${ }^{1,2^{*}}$
}

\author{
* Correspondence: diana. \\ filip@econ.ubbcluj.ro \\ 'Department of Statistics, \\ Forecasting and Mathematics, \\ Faculty of Economics and Business \\ Administration, University Babeş \\ Bolyai, 400591 Cluj-Napoca, \\ Romania \\ Full list of author information is \\ available at the end of the article
}

\begin{abstract}
For models of majority voting over fixed-income taxations, we mathematically define the concept of least core. We provide a sufficient condition on the policy space such that the least core is not empty. In particular, we show that the least core is not empty for the framework of quadratic taxation, respectively piecewise linear tax schedules. For fixed-income quadratic taxation environments with no Condorcet winner, we prove that for sufficiently right-skewed income distribution functions, the least core contains only taxes with marginal-rate progressivity.
\end{abstract}

\section{Introduction}

The literature of the positive theory of income taxation regards the tax schemes in democratic societies as emerging, explicitly or implicitly, from majority voting (see Romer [1,2], Roberts [3], Cukierman and Meltzer [4], Marhuenda and Ortuño-Ortin $[5,6])$. A very important mathematical difficulty related to this view is that the existence of a Condorcet majority winner is not guaranteed, since the policy space of tax schedules is usually multidimensional (see for example Hindriks [7], Grandmont [8], Marhuenda and Ortuño-Ortin [6], Carbonell and Ok [9]).

The possible inexistence of a Condorcet winner can be regarded as predicting political instability with respect to the taxation system to be agreed on. However, the stability of tax schedules in democratic societies is already a well-established stylized fact (see Grandmont [8], Marhuenda and Ortuño-Ortin [6]). As noted by Grandmont [8], possible ways out followed in the literature imply restricting to flat taxes (Romer [1], Roberts [3]), or to quadratic taxations and some tax to be ideal for some voter (Cukierman and Meltzer [4]), introducing uncertainty about the tax liabilities of a new proposal (Marhuenda and Ortuño-Ortin [6]), considering solution concepts less demanding than the core (De Donder and Hindriks [10]).

In a majority game in coalitional form of voting over income distributions, Grandmont [8] proves the usual result that the core is empty (no majority Condorcet winner). Also the solution concept of the least core implies no insights, since it contains just the egalitarian income distribution, in case it is not empty. Therefore, the author explores two variants of the bargaining set in order to understand the apparent stability of tax schedules in democratic societies. Grandmont [8] argues that in his setup, voting over tax schemes is equivalent to voting directly over income distributions.

(C) 2011 Curt et al; licensee Springer. This is an Open Access article distributed under the terms of the Creative Commons Attribution License (http://creativecommons.org/licenses/by/2.0), which permits unrestricted use, distribution, and reproduction in any medium, provided the original work is properly cited. 
However, most of the literature imposes some fairness principles to the tax schedules, i.e., a tax is increasing with the revenues in such a way that it does not change the post-tax income ranking (see Marhuenda and Ortuño-Ortin [5], Roemer [11], Hindriks [7], Carbonell and Klor [12], De Donder and Hindriks [10], Carbonell and Ok [9]). Moreover, a tax is not necessarily purely redistributive (Marhuenda and OrtuñoOrtin [5], Carbonell and Ok [9]). Therefore, even if keeping the feature that a tax is not distortionary, voting in the above-mentioned taxation models is not equivalent with voting over income distributions as in Grandmont [8]. Consequently, despite the fact that the core in such setups is empty, the analysis of the least core may provide more than trivial results on the stability, as well as on the prevalence of the marginalrate progressivity in income taxation. (The latter is one important question that the positive theory of income taxation tries to answer, see Marhuenda and Ortuño-Ortin [5,6], Roemer [11], Hindriks [7], Carbonell and Klor [12], De Donder and Hindriks [10], Carbonell and Ok [9], among many others.)

The contribution of this article is that it defines and analyzes the general properties of the least core in fixed-income taxation models. Theorem 1 provides a necessary condition on the policy space $U$ to have at least one tax in the least core, for the case of (absolutely) continuous income distribution functions. Propositions 2 and 3 prove that the least core is not empty for the framework of quadratic taxations, respectively picewise linear tax schedules. In Theorem 2, we show that for fixed-income quadratic taxation environments with no Condorcet winner, and for sufficiently right-skewed income distribution functions, the least core is characterized by taxes with marginalrate progressivity. This result seems in line with the heuristic argument commonly invoked to explain the prevalence of the marginal-rate progressivity, that is, the number of relatively poor (self-interest) voters exceeds that of richer ones. The result also argues in favor of the fact that analyzing the least core in particular fixed-income taxation models can provide useful insights on the major questions of the positive theory of income taxation.

\section{The model}

\subsection{General setup}

The economy consists of a large number of individuals who differ in their (fixed) income. Each individual is characterized by her income $x \in[0,1]$. The income distribution can be described by a function $F:[0,1] \rightarrow[0,1]$, continuous and differentiable almost everywhere and increasing on the interval $[0,1]$. Each individual with income $x$ $\in[0,1]$ has strictly increasing preferences on the set of her possible net incomes. The associated Lebesque-Stieltjes probability measure induced by $F$ is denoted by $v(S)$ and $v(S)=\int_{S} \mathrm{~d} F(x)$ for any Lebesque-Stieltjes measurable set $S \subseteq[0,1]$. The fixed amount $0 \leq R<\bar{y}=\int_{[0,1]} \mathrm{d} F(x)$ should be collected through means of a tax imposed on the agents. ${ }^{a}$ When $R=0$, the tax is purely redistributive. It is assumed that there is no tax evasion, respectively there are no distortions induced by the taxation system in the economy. In one word, the pre-tax income is fixed (in the sense that it is given and not influenced by the taxation system).

A set of admissible tax schedules $U=U(F, R)$ contains functions $t$ continuous on [0, 1] that necessarily satisfy, for a given $F$ and $R$, the following conditions ${ }^{b}$ : 


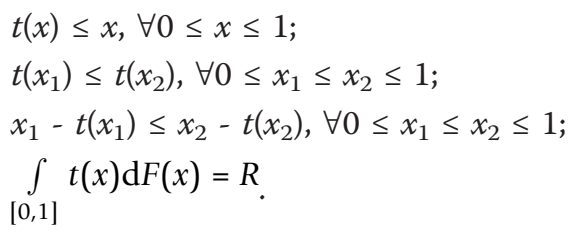

It is noteworthy that the continuity of $t$ is actually implied by the conditions (2) and (3). Moreover, the tax functions that satisfy the conditions (1)-(4) are uniformly bounded by the constant 1. A tax schedule $t$ is (marginally) progressive (regressive) if and only if $t(x)$ is convex (concave).

In the following, we present examples of restricted policy spaces $U$ of income tax functions, which, as underlined in the introduction, were used in the literature of the positive theory of income taxation to provide useful insights to specific questions of this field.

\section{Example 1 (quadratic tax functions)}

Consider quadratic functions of the form $t:[0,1] \rightarrow(-\infty, 1], t(x)=a x^{2}+b x+c$. The set of quadratic tax functions that satisfy the feasibility conditions (1)-(4) is denoted by $Q T=Q T(F, R)$. It can be easily proved that conditions (1)-(4) restrict the set of feasible taxes to $t:[0,1] \rightarrow[-1,1], t(x)=a x^{2}+b x+c$, where $0 \leq b \leq 1,0 \leq 2 a+b \leq 1$, and $c$ $\leq 0$. According to condition (4), we can express $c$ as a function of $a$ and $b$. Indeed, we have: $\quad R=\int_{[0,1]}\left(a x^{2}+b x+c\right) \mathrm{d} F(x)=a\left(\sigma^{2}+\bar{y}^{2}\right)+b \bar{y}+c, \quad$ wherefrom $c=R-a \bar{y}_{2}-b \bar{y} \leq 0$ and $\sigma^{2}$ is the variance of the income distribution. In conclusion, the feasible conditions, denoted with $\left(F A_{1}\right)$, for a quadratic tax function $t:[0,1] \rightarrow[-1,1], t(x)=a x^{2}+b x+R-a \bar{y}_{2}-b \bar{y}$ are as follows:

$$
\left(F A_{1}\right)\left\{\begin{array}{l}
0 \leq b \leq 1 \\
0 \leq 2 a+b \leq 1 \\
a \bar{y}_{2}+b \bar{y} \geq R
\end{array}\right.
$$

\section{Example 2 (piecewise linear tax functions)}

Let $m \geq 2$ be a natural number and let $x_{j}, j=0, \ldots, m$, be $m+1$ fixed real numbers that satisfy the following inequalities: $0=x_{0}<x_{1}<\ldots<x_{m-1}<x_{m}=1$. We consider $\mathrm{PWT}=\operatorname{PWT}(F, R)$, the set of $m$-bracket piecewise linear tax functions that satisfy the feasibility conditions (1)-(4) and change their definition expression at the points $x_{j}, j=$ $1, \ldots, m-1$. It can be easily proved that conditions (1)-(4) restrict the set of $m$-bracket piecewise linear feasible taxes to functions of the form:

$$
t:[0,1] \rightarrow[-1,1], t(x)=\left\{\begin{array}{cc}
a_{1} x+b_{1}, & x \in\left[0, x_{1}\right) \\
a_{2} x+b_{2}, & x \in\left[x_{1}, x_{2}\right) \\
\ldots & \\
a_{m} x+b_{m}, & x \in\left[x_{m-1}, 1\right]
\end{array}\right.
$$

, which satisfy the following conditions, denoted with $\left(F A_{2}\right)$ :

$$
\left(F A_{2}\right)\left\{\begin{array}{l}
0 \leq a_{j} \leq 1, \quad \text { for each } j=1, \ldots, m \\
a_{j} x_{j}+b_{j}=a_{j+1} x_{j}+b_{j+1}, \quad \text { for each } j=1, \ldots, m-1 \\
\left(1-a_{j}\right) x_{j-1} \geq b_{j}, \quad \text { for each } j=1, \ldots, m, \quad \text { and }\left(1-a_{m}\right) \geq b_{m} \\
\sum_{j=1}^{m} a_{j} \int x \mathrm{~d} F(x)+\sum_{j=1}^{m} b_{j}\left[F\left(x_{j-1}\right)-F\left(x_{j}\right]\right.
\end{array}\right.
$$




\section{Remark on Example 2}

Note that the first condition above guarantees that every tax and every post-tax function are increasing, the second condition shows that all considered tax functions are continuous, the third condition guarantees that the tax payed by each agent is smaller than the corresponding pre-tax income, and the forth condition assures that the collected tax from the agents is $R$. Note as well that if $2 \leq k \leq m$ then the class PWT also contains $k$-bracket piecewise linear tax functions (that satisfy the conditions (1)-(4)) that change their definition expression at $k-1$ points out of the set $\left\{x_{1}, \ldots, x_{m-1}\right\}$. We mention that a $m$-bracket piecewise linear tax $t$ is progressive if $a_{1} \leq a_{2} \leq \ldots \leq a_{m}$ and regressive if conversely $a_{1} \geq a_{2} \geq \ldots \geq a_{m}$.

\subsection{Condorcet majority winner, core, $\varepsilon$-core, and least core}

Given a set $U$ of admissible tax schedules and a function $t \in U$, a tax policy $q \in U$ is an objection to $t$ if $v\{x \in[0,1]: q(x)<t(x)\}>v\{x \in[0,1]: q(x)>t(x)\}$. That means $v\{x$ $\in[0,1]: q(x) \leq t(x)\}>1 / 2$, thus the tax $q$ is (weakly) preferred by a majority of individuals to the $\operatorname{tax} t$. A tax function $t \in U$ is a Condorcet majority winner if and only if there is no objection to it, meaning that it is preferred by a majority of individuals to any other feasible tax. We denote by $\mathrm{Obj}_{U}(t)$ the set of all objections to the taxation $t$. Therefore, the above definitions for $t$ being a Condorcet winner are equivalent to the condition $\operatorname{Obj}_{U}(t)=\varnothing$. In the corresponding majority game over taxes in coalitional form, the set of all Condorcet winners represents the core and the inexistence of a Condorcet majority winner is equivalent to the fact that the core is empty (see Grandmont [8]).

Given $t, q \in U$, the scalar $d(t, q)=\int_{\{x \in[0,1]: q(x)<t(x)\}}(t(x)-q(x)) \mathrm{d} F(x)$ represents the total gain of those individuals that are better off if the tax schedule changes from $t$ to $q$. Because both taxes collect the same amount, the other interpretation is that $d(t, q)$ represents the total loss of those individuals that are worse off if the tax schedule changes from $t$ to $q$. The value $d(t, q)$ is equal to $d(q, t)=\int_{\{x \in[0,1]: t(x)<q(x)\}}(q(x)-t(x)) \mathrm{d} F(x)$ and it is equal as well with $1 / 2 \int_{[0,1]}|t(x)-q(x)| \mathrm{d} F(x)$. It should be noted that $d$ is a metric that is the restriction to the tax function space $U$ of the $L^{1}$ metric: $\|t-q\|_{1}=\int_{[0,1]}|t(x)-q(x)| \mathrm{d} v(x)=\int_{[0,1]}|t(x)-q(x)| \mathrm{d} F(x)$ on the measurable space $([0$, $1], v)$. Since in $L^{1}([0,1], v), t=q$ if and only if $t(x)=q(x)$ a.e., the same convention applies to the space of interest $U$. This convention also subscribes to a certain economic logic. In any voting game, either in a coalitional setup or a non-cooperative one, the behavior of tax schedules on those income intervals that are represented by zero measure groups of individuals does not have any influence on the final outcome of the game.

Given $\varepsilon>0$, the set $C(\varepsilon)$ contains all the taxes for which there is no objection such that the total gain of the better off agents under the objection is strictly greater than $\varepsilon$. In the simple majority game in coalitional form associated to our setup, the set $C(\varepsilon)$ is the $\varepsilon$-core. It contains those taxes for which it is impossible to find objections such that the supporting coalition remains strictly better off even after paying the cost $\varepsilon$ of forming it.

In Grandmont [8], a way to understand the stability of a status quo income distribution is to be in all $\varepsilon$-cores, $\varepsilon>0$, whenever they are not empty (i.e., the least core, as in Einy et al. [13]). Similarly, we define here the set $\bigcap_{\{\varepsilon>0: C(\varepsilon) \neq \emptyset\}} C(\varepsilon)$. Within a static 
coalitional framework, Litan [14] argues that this is a concept of taxation stability. He also discusses some directions to establish the non triviality of the concept in income taxation environments with non-distortionary taxes.

In this article, in the results section, we analyze the general properties of the least core, and under what conditions this set is not empty in fixed-income taxation models. We analyze as well the implications of the concept for the quadratic taxation model. This is among the models that are very used in the literature to provide powerful insights on the questions raised by the positive theory of income taxation (see Hindriks [7], De Donder and Hindriks [10,15], Cukierman and Meltzer [4], etc.).

\section{Results}

\subsection{Some properties and the non triviality of the least core in fixed-income taxation environments}

The next proposition states two important properties of the least core, as defined in our general taxation setup. First, in the case the core is not empty, then the least core reduces to the core concept. Second, the taxes in the least core can be found by solving a min sup problem expressed in terms of the distance $d$. These results are in line with properties that the least core has, when it is defined for discrete policy spaces (see Einy et al. [13]).

Proposition 1. Let $U$ be a set of tax functions that satisfy the conditions (1)-(4). If the set $\bigcap_{\{\varepsilon>0: C(\varepsilon) \neq \emptyset\}} C(\varepsilon)$ is not empty then the following assertions are true:

If we denote by $\underline{\varepsilon}=\inf _{\{\varepsilon>0: C(\varepsilon) \neq \emptyset\}} \varepsilon$, then $\bigcap_{\{\varepsilon>0: C(\varepsilon) \neq \varnothing\}} C(\varepsilon)=C(\underline{\varepsilon}) \underline{\varepsilon}=\inf _{t \in U} \sup _{q \in O \mathrm{Obj}_{U}(t)} d(t, q)$

$\underline{\varepsilon}=0$ if and only if $\bigcap_{\{\varepsilon>0: C(\varepsilon) \neq \emptyset\}} C(\varepsilon)$ is the set of Condorcet majority winnersinf $_{t \in U} \sup _{q \in O \mathrm{Obj}_{U}(t)} d(t, q)=\min _{t \in U} \sup _{q \in \mathrm{Obj}_{U}(t)} d(t, q)$

Proof. We note that all the supremums and infimums of $d(t, q)$ are taken over subsets of $\mathbb{R}^{+}$, hence the supremum over the empty set is 0 and the infimum over the empty set is $\infty$.

(i), (ii) The proofs can be left to the reader since they are immediate consequences of the definitions of infimum and supremum of a given set.

(iii) Suppose first that $\underline{\varepsilon}=0$. We have to prove (see (i)) that $C(0)$ coincides to the set of all Condorcet winners. Since it is obvious that every Condorcet winner $t$ belongs to $C(0)$ (due to the convention made above: $\sup _{q \in \varnothing} d(t, q)=0$ ), it remains to show that every function in $C(0)$ is a Condorcet winner. Suppose by contrary, that there is $t \in C(0)$ such that $\operatorname{Obj}_{U}(t) \neq \varnothing$. For $t \in C(0)$ and $q \in \mathrm{Obj}_{U}(t)$ the distance $d(t . q)$ is 0 wherefrom we get that $t \stackrel{\text { a.e. }}{=} q$, which is a contradiction with $q \in \operatorname{Obj}_{u}(t)$.

Suppose now that $C(\underline{\varepsilon})$ is the set of all Condorcet majority winners. In order to prove that $\underline{\varepsilon}=\inf _{\{\varepsilon>0: C(\varepsilon) \neq \emptyset\}} \varepsilon=0$ it is sufficient to prove that for every $\varepsilon>0$ the set $C(\varepsilon)$ is not empty, which is obviously true, due to the inclusion $C(\varepsilon) \supseteq C(0) \neq \varnothing$.

(iv) It is left to the reader, being an immediate consequence of the definitions and of the hypothesis that $C(\underline{\varepsilon}) \neq \varnothing$.

For the next theorem and throughout the rest of the section, we will assume that every distribution function $F$ that generates a Lebesque-Stieltjes measure is absolutely continuous, hence it has a density that is the a.e. derivative with respect to the Lebesgue measure on $[0,1], \lambda$, of the given distribution function. Also, we suppose that the 
density function is almost everywhere continuous with respect to $\lambda$. It should be noticed that many distribution functions used to model the repartition of income among the individuals of a society have the required properties (see for instance the beta distributions in De Donder and Hindriks [10,15], or the examples of income distribution functions from Carbonell and Ok [9]). The next theorem provides a necessary condition on the policy space $U$ to have at least one tax in the least core, for the case of (absolutely) continuous income distribution functions.

Theorem 1. Let $U$ be a set of tax functions that satisfy the conditions (1)-(4). If the set $U$ is complete with respect to metric $d$, then $\bigcap_{\{\varepsilon>0: C(\varepsilon) \neq \emptyset\}} C(\varepsilon)$ is not empty.

Proof. Remember that the metric $d$ is the restriction to the tax function space $U$ of the $L^{1}$ metric: $\|t-q\|_{1}=\int_{[0,1]}|t(x)-q(x)| \mathrm{d} v(x)=\int_{[0,1]}|t(x)-q(x)| \mathrm{d} F(x)$ on the measurable space $([0,1], v)$. Moreover, since $F$ is an absolutely continuous function, we also have $d(t, q)=\int_{[0,1]}|t(x)-q(x)| F^{\prime}(x) \mathrm{d} \lambda(x)$.

The conclusion of the theorem can be obtained by applying the well-known result that asserts that in any topological compact space, any family of closed subsets with the finite intersection property has non-empty intersection (see Edwards [[16], p. 17]). We apply the above-mentioned result for the metric space $(U, d)$ and the family of sets: $\{C(\varepsilon)\}_{\{\varepsilon>0: C(\varepsilon) \neq \emptyset\} \text {. }}$

We start by proving that for each $\varepsilon>0$ such that $C(\varepsilon) \neq \varnothing, C(\varepsilon)$ is a closed subset of $(U, d)$. For this, let $t \in \overline{C(\varepsilon)} \subset \bar{U}=U$ (the previous equality is true because any complete subspace of a metric space is closed). Since $t \in \overline{C(\varepsilon)}$, there exists a sequence $\left(t_{n}\right)_{n}$ $\subseteq C(\varepsilon)$ such that $t_{n} \stackrel{L^{1}}{\rightarrow} t$. From the $L^{1}$ convergence of the $\left(t_{n}\right)_{n}$ sequence of taxes results the existence of a subsequence $\left(t_{n_{k}}\right)_{k} \subseteq\left(t_{n}\right)_{n}$ such that $t_{n_{k}} \stackrel{\text { a.e. }}{\rightarrow} t$. (see Ash [[17], pp. 9293, Theorems 2.5.1 and 2.5.3]). Let $M \subset[0,1]$ be the set for which $v(M)=1,(v([0,1]$ $(M)=0)$ and $t_{n_{k}}(x) \rightarrow t(x)$ for any $x \in M$.

In order to prove that $t \in C(\varepsilon)$ it is sufficient to show that $d(t, q) \leq \varepsilon$ for each $q \in$ $\operatorname{Obj}_{U}(t)$. Let $q \in \operatorname{Obj}_{U}(t)$. Then, $v(A)>1 / 2$, where $A=\{x \in[0,1]:(q-t)(x)<0\}$. In the following, we shall prove that there exists $k_{0} \in \mathbb{N}$ such that $q$ is an objection to $t_{n_{k}}$ for any $k>k_{0}$. For this, it is sufficient to show that there exists $k_{0} \in \mathbb{N}$ such that $v\left(A_{n_{k}}\right)>1 / 2$, where $A_{n_{k}}=\left\{x \in[0,1]:\left(q-t_{n_{k}}\right)(x)<0\right\}$. The previous statement results as a straightforward consequence of the Lebesque's dominated convergence theorem applied to the sequence of measurable functions $\left\{\chi_{A_{n_{k}} \cap A}\right\}_{k}=\left\{\chi_{A_{n_{k}}} \chi_{A}\right\}_{k}$, dominated by the constant unit function on the finite measure space $L^{1}([0,1], v)$. We check now that all the conditions of the Lebesque dominated convergence theorem are fulfilled. The measurability conditions are trivially fulfilled by the involved functions. For the almost everywhere convergence consider $x \in M$. If $x \in A \cap M$, since $\lim _{k \rightarrow \infty}\left(q-t_{n_{k}}\right)(x)=(q-t)(x)<0$, it results that there exists, $k^{\prime} \in \mathbb{N}$ such that for every $k \geq k^{\prime}$ we have $\left(q-t_{n_{k}}\right)(x)<0$, i.e, $x \in A_{n_{k}}$. It follows that if $x \in M \cap A$ then $\chi_{A_{n_{k}}}(x)=\chi_{A}(x)=1, k \geq k^{\prime}$, which implies $\chi_{A_{n_{k}} \cap A}(x) \rightarrow \chi_{A}(x)$. If $x \in([0,1] \backslash A) \cap M$ then $\chi_{A_{n_{k}} \cap A}(x)=\chi_{A}(x)=0$, hence $\chi_{A_{n_{k}} \cap A}(x) \rightarrow \chi_{A}(x)$. By applying the Lebesque's dominated convergence theorem, we get $\int_{[0,1]} \chi_{A_{n_{k}} \cap A}(x) \mathrm{d} F(x) \rightarrow \int_{[0,1]} \chi_{A}(x) \mathrm{d} F(x)$ wherefrom $v\left(A_{n_{k}}\right) \geq v\left(A_{n_{k}} \cap A\right) \rightarrow v(A)>1 / 2$. It follows that there exists $k_{0} \in \mathbb{N}$ such that for any $k \geq k_{0}$, we have $v\left(A_{n_{k}}\right)>1 / 2$ and in consequence $q$ is an objection to $t_{n_{k}}$, for each 
$k \geq k_{0}$, so $d\left(q, t_{n_{k}}\right) \leq \varepsilon$. Hence, $d(q, t) \leq d\left(q, t_{n_{k}}\right)+d\left(t_{n_{k}}, t\right) \leq \varepsilon+d\left(t_{n_{k}}, t\right)$. Taking the limit after $k \rightarrow \infty$, we obtain that $d(t, q) \leq \varepsilon$ as desired.

Since for each $\varepsilon_{1}<\varepsilon_{2}$, we have $C\left(\varepsilon_{1}\right) \subseteq C\left(\varepsilon_{2}\right)$, then $\{C(\varepsilon)\}_{\{\varepsilon>0: C(\varepsilon) \neq \varnothing\}}$ is a family of closed sets, which has the finite intersection property.

It remains for us only to justify the compactness of $U$. Since $U$ is closed, it is sufficient to show that $U$ is relatively compact in $\left(L^{1},\|\cdot\|_{1}\right)$ (meaning that it's closure is compact). For this, we apply the following variation (see Simon [[18], p. 74]) of Kolmogorov-Riesz-Fréchet theorem (the " $L^{p}$-version" of the Ascoli-Arzela theorem):

The set $G$ is relatively compact in $L^{1}([0,1], \lambda)$ if and only if:

(i)There is $0 \leq a_{1}<a_{2} \leq 1$ such that $\int_{\left[a_{1}, a_{2}\right]} g(x) \mathrm{d} \lambda(x)$ is bounded uniformly for $g \in$ G.

(ii) $\int_{[0,1-h]}|g(x+h)-g(x)| \mathrm{d} \lambda(x) \rightarrow 0$ as $h \rightarrow 0$ uniformly for $g \in G$.

We apply the previous result for $G=\left\{t F^{\prime}: t \in U\right\} \subset L^{1}([0,1], \lambda)$.

The conditions from the above mentioned result are fulfilled, due to the properties of the tax functions. Indeed, if we take $a_{1}=0$ and $a_{2}=1$ then for each $t \in U$, we have $\int_{[0,1]} t(x) F^{\prime}(x) \mathrm{d} \lambda(x)=\int_{[0,1]} t(x) \mathrm{d} v(x)=R$. Therefore, the condition (i) is fulfilled. For $f$ $\in U$, by using the properties (1), (3), and the uniform boundness of the tax functions, we

$\leq h F(1-h)+\int_{[0,1-h]}\left|F^{\prime}(x+h)-F^{\prime}(x)\right| \mathrm{d} \lambda(x) \rightarrow 0$ -

$\leq h F(1-h)+\int_{[0,1-h]}\left|F^{\prime}(x+h)-F^{\prime}(x)\right| \mathrm{d} \lambda(x) \rightarrow 0$ -

$\leq h F(1-h)+\int_{[0,1-h]}\left|F^{\prime}(x+h)-F^{\prime}(x)\right| \mathrm{d} \lambda(x) \rightarrow 0$, as $h \rightarrow 0$. The convergence to 0 of the previous integral is a straightforward application of the Lebesque's convergence theorem for the sequence of functions defined by: $\left|F^{\prime}\left(x+h_{n}\right)-F^{\prime}(x)\right|$, if $x \in\left[0,1-h_{n}\right]$, and 0 , if $x \in\left[1-h_{n}, 1\right]$. In consequence, $G$ is relatively compact in $\left(L^{1}[0, \lambda],\|\cdot\|_{1}\right)$ and hence $U$ is relatively compact in $\left(L^{1}[0, v],\|\cdot\|_{1}\right)$, as required.

Notice that Theorem 1 does not say anything about the cardinality of the least core. In fact, there may be cases in which the cardinality is not finite. However, as it can be seen in the next subsections, the theorem insures that in many instances in which the core is empty, the least core is actually not (for example the quadratic taxation case, or the piecewise linear taxation case). Once the non-emptiness of least core is established, only then the analysis of its structure can be performed.

\subsection{Least core non triviality for quadratic and piecewise linear taxes}

As already mentioned, the framework of quadratic taxations represents a workhorse model, providing useful insights into the specific questions of the positive theory of income taxation. The quadratic taxation model was first used by Cukierman and Meltzer [4], then Roemer [11], and subsequently by Hindriks [7], De Donder and Hindriks $[10,15]$ to derive interesting results. The next proposition has as direct corollary the fact that for quadratic taxations our analyzed setup has a non-empty least core.

Proposition 2. Let $Q T(F, R)=Q T$ be the set of quadratic tax functions defined in Example 1. Then $(Q T, d)$ is complete.

Proof. Consider a Cauchy sequence $\left\{t_{n}\right\}_{n \geq 1}$ in $(Q T, d)$. Suppose that $t_{n}(x)=a_{n}\left(x^{2}-\bar{y}_{2}\right)+b_{n}(x-\bar{y})+R, x \in[0,1]$. Since $\left\{t_{n}\right\}_{n \geq 1}$ is a Cauchy sequence in the 
complete metric space $\left(L^{1}[0,1], d\right)$, it will be convergent to some $t \in L^{1}[0,1]$. Since the convergence $t_{n} \stackrel{L^{1}}{\rightarrow} t$ implies the a.e. convergence to $t$ of a subsequence of the given sequence (without loss of generality we can denote the a.e. convergent subsequence by $\left.\left\{t_{n}\right\}_{n \geq 1}\right)$, there exist at least two distinct points, $x_{1} \neq x_{2}$, such that $\lim _{n \rightarrow \infty} t_{n}\left(x_{i}\right)=t\left(x_{i}\right)$, $i=\overline{1,2}$. Due to the convergence of the sequences $\left\{t_{n}\left(x_{i}\right)\right\}_{n \geq 1}, i=\overline{1,2}$, and of the fact that $x_{1} \neq x_{2}$, it results the convergence of the sequences $\left(a_{n}\right)_{n}$ and $\left(b_{n}\right)_{n}$. If $a$ and $b$ are the limits of these sequences, then for every $x \in[0,1]$ we have $\lim _{n \rightarrow \infty} t_{n}(x)=\lim _{n \rightarrow \infty}\left[a_{n}\left(x^{2}-\bar{y}_{2}\right)+b_{n}(x-\bar{y})+R\right]=a\left(x^{2}-\bar{y}_{2}\right)+b(x-\bar{y})+R \stackrel{n o t .}{=} \bar{t}(x)$. The feasibility conditions $\left(F A_{1}\right)$ for the function $\bar{t}$ are easy consequences of the similar properties of the tax functions $t_{n}, n \in \mathbb{N}$, hence $\bar{t} \in Q T$. Because $t_{n} \stackrel{L^{1}}{\rightarrow} t$ and $\bar{t} \stackrel{\text { a.e. }}{=} t$, we get that $t_{n} \stackrel{L^{1}}{\rightarrow} \bar{t}$. Therefore $(Q T, d)$ is complete.

The framework of piecewise linear taxations was used in the literature to analyze questions regarding the preponderant marginal-rate progressive taxations in democracies (see for instance Carbonell and Klor [12] and Klor [19]). The next proposition has as direct corollary the fact that for piecewise linear taxations, our analyzed setup has a non-empty least core.

Proposition 3. Let $m \geq 2$ and $P W T(F, R)=P W T$ be the set of $m$-bracket piecewise tax functions defined in Example 2. Then, (PWT, $d)$ is complete.

Proof. Consider a Cauchy sequence $\left\{t_{n}\right\}_{n \geq 1}$ in $(P W T, d)$. Suppose that $t_{n}(x)=a_{j}^{n} x+b_{j}^{n}$, $x \in\left(x_{j-1}, x_{j}\right], j=\overline{1, m}$. Since $\left\{t_{n}\right\}_{n \geq 1}$ is a Cauchy sequence in the complete metric space $\left(L^{1}[0,1], d\right)$, it will be convergent to some $t \in L^{1}[0,1]$. The $L^{1}$ convergence implies the a.e. convergence to $t$ of a subsequence of the given sequence. Without loss of generality we can denote the a.e. convergent subsequence by $\left\{t_{n}\right\}_{n \geq 1}$.

If $j \in\{1, \ldots, m\}$ is such that $v\left(x_{j-1}, x_{j}\right]>0$, then there exist at least two distinct points in $\left(x_{j-1}, x_{j}\right], x_{1}^{j} \neq x_{2}^{j}$, such that $\lim _{n \rightarrow \infty} t_{n}\left(x_{i}^{j}\right)=t\left(x_{i}^{j}\right), i=\overline{1,2}$. Due to the convergence of the sequences $\left\{t_{n}\left(x_{i}^{j}\right)\right\}_{n \geq 1}, i=\overline{1,2}$, and of the fact that $x_{1}^{j} \neq x_{2}^{j}$, it results the convergence of the sequences $\left(a_{j}^{n}\right)_{n}$ and $\left(b_{j}^{n}\right)_{n}$. If $a_{j}$ and $b_{j}$ are the limits of these sequences, then for each $x \in\left(x_{j-1}, x_{j}\right]$, we have $\lim _{n \rightarrow \infty} t_{n}(x)=\lim _{n \rightarrow \infty}\left(a_{j}^{n} x+b_{j}^{n}\right)=a_{j} x+b_{j} \stackrel{\text { not. }}{=} \bar{t}(x)$.

If $j \in\{1, \ldots, m\}$ is such that $v\left(x_{j-1}, x_{j}\right]=0$, then for every $g \in L^{1}[0,1]$, we have $\int_{\left[x_{j-1}, x_{j}\right]} g(x) \mathrm{d} F(x)=0$. In this case, if $j \neq 1$ and $j \neq m$, we define the function $\bar{t}$ on $\left[x_{j-1}\right.$, $x_{j}$ ] to be the linear function whose graph is the segment that connects in the plane the points $\left(x_{j-1}, a_{j-1} x_{j-1}+b_{j-1}\right)$ to $\left(x_{j}, a_{j+1} x_{j}+b_{j+1}\right)$. For $j=1$ or $j=m$, the graph of $\bar{t}$ connects the points $(0.0)$ and $\left(x_{1}, a_{2} x_{1}+b_{2}\right)$, respectively, $\left(x_{m-1}, a_{m-1} x_{m-1}+b_{m-1}\right)$ and (1, 1).

The feasibility conditions $\left(F A_{2}\right)$ for the function $\bar{t}$ are easy consequences of the properties of the tax functions $t_{n}, n \in \mathbb{N}$, hence $\bar{t} \in P W T$. Because $t_{n} \stackrel{L^{1}}{\rightarrow} t$ and $\bar{t} \stackrel{\text { a.e. }}{=}$, we get that $t_{n} \stackrel{L^{1}}{\rightarrow} \bar{t}$. Therefore $(P W T, d)$ is complete.

\subsection{Marginal progressivity and the least core in fixed-income quadratic taxation environments}

De Donder and Hindriks [15] and Curt et al. [20] provide a complete mathematical description of those fixed-income distributions for which a majority winning tax exists (or does not exist), in the quadratic taxation model à la Roemer [11], with tax 
schedules that are purely redistributive. Curt et al. [21] analyze the same problem for tax schedules that are not purely redistributive. For income distributions with the median less than the mean, in case a Condorcet winner exists then it implies maximum marginal progressivity. In the next theorem, we prove that, when a Condorcet winner does not exist, for sufficiently right-skewed income distribution functions, the least core is characterized by marginal progressivity as well (however, not necessarily maximal). The proof is for purely redistributive taxations, however it can be adapted for tax schedules that are not purely redistributive.

We introduce first some notation, according to Curt et al. [21]. Let $h:[0,1] \rightarrow \mathbb{R}, h(x)=u x^{2}+v x-u \bar{y}_{2}-v \bar{y}, u \in \mathbb{R}^{*}, v \in \mathbb{R}$, and let $\alpha=-\frac{v}{2 u \bar{y}}$. Then, for each $\alpha \in \mathbb{R}$, the quadratic function $h$ has two real roots $x_{1}(\alpha)=\alpha \bar{y}-\sqrt{(\alpha-1)^{2} \bar{y}^{2}+\sigma^{2}}$ and $x_{2}(\alpha)=\alpha \bar{y}+\sqrt{(\alpha-1)^{2} \bar{y}^{2}+\sigma^{2}}$, which vary as functions of $\alpha$. The conditions on the income distribution function for the existence/ non existence of a majority winning tax are expressed in terms of $x_{1}(\alpha)$ and $x_{2}(\alpha)$ (see De Donder and Hindriks [15], Curt et al. [20,21]).

Theorem 2. Let $F$ be a distribution function such that $1-\sqrt{(1-\bar{\gamma})^{2}+\sigma^{2}}<\gamma_{m}<\bar{\gamma}$. If $F\left(\frac{\bar{\gamma}_{2}}{\bar{y}}\right)-F\left(\frac{\bar{y}-\bar{y}_{2}}{1-\bar{y}}\right)<\frac{1}{2}$ and there is $\alpha_{0} \in\left(\frac{1}{2 \bar{y}^{\prime}}, \frac{1-\bar{y}_{2}}{2 \bar{y}(1-\bar{y})}\right)$ such that $F\left(x_{2}\left(\alpha_{0}\right)\right)-F\left(x_{1}\left(\alpha_{0}\right)\right)<\frac{1}{2}$, then the core is empty (there is no Condorcet majority winner).

If in addition to the above conditions, $F\left(x_{2}(\alpha)\right)-F\left(x_{1}(\alpha)\right)>1 / 2$ for each $\alpha \in\left(\frac{\bar{y}_{2}}{\bar{y}}, \frac{1}{2 \bar{y}}\right]$, then the set $\bigcap_{\{\varepsilon>0: C(\varepsilon) \neq \emptyset\}} C(\varepsilon)$ contains only progressive tax functions.

Proof. The proof of item (i) can be found in Curt et al. [20]. We prove below item (ii).

For each tax function $t=(a, b)$, we shall determine and represent geometrically the feasibility area $F A=\left\{(u, v): u=\bar{a}-a, v=\bar{b}-b, q=(\bar{a}, \bar{b}) \in \operatorname{Obj}_{\mathrm{QT}}(t)\right\}$.

From the feasibility conditions $\left(F A_{1}\right)$ for the objection function $q$, we obtain that the coefficients $u$ and $v$ must satisfy the inequalities: $-b \leq v \leq 1-b,-(2 a+b) \leq 2 u+v \leq 1$ - $(2 a+b)$, and $u \bar{y}_{2}+v \bar{y} \geq-\left(a \bar{y}_{2}+b \bar{y}\right)$. Hence the geometric representation (see Figures 1 and 2) will be the interior and the sides of the parallelogram whose vertices are $A, B$, $C, D$ (for a regressive tax function) or $A^{\prime}, B^{\prime}, C^{\prime}, D^{\prime}$ (for a progressive tax function). We remark that the budget constraint condition generates a line that passes through $A$ (respectively $A^{\prime}$ ) and the vertices $B, C$, and $D$ (respectively $B^{\prime}, C^{\prime}$ and $D^{\prime}$ ) are situated above the budget line.

Next, we shall deduce the expression of the distance $d(q, t), q \in \operatorname{Obj}_{\mathrm{QT}}(t)$, as a function of $u, v$, and $\alpha=-v /(2 u \bar{y})$.

We analyze first the case when $u \neq 0$.

- For $\alpha \in\left(\infty, \bar{y}_{2} /\left(2 \bar{y}^{2}\right)\right)$ since (see Lemma 1 in Curt et al. [21]) $x_{1}(\alpha) \leq 0<y_{m}<\bar{y} \leq x_{2}(\alpha)<1$, we get that $q=(\bar{a}, \bar{b}) \in \operatorname{Obj}_{\mathrm{QT}}(t)$ for each $(\bar{a}, \bar{b})$ for which $(u, v)=(\bar{a}-a, \bar{b}-b) \in F$ and $u>0$. Elementary computations give us $d(q, t)=u \int_{\left[0, x_{2}(\alpha)\right]} h(x, \alpha) \mathrm{d} F(x)$, where $h(x, \alpha)=-x^{2}+2 \alpha \bar{y} x+\bar{y}_{2}-2 \alpha \bar{y}^{2}$.

- For $\alpha \in\left(\bar{y}_{2} /\left(2 \bar{y}^{2}\right),\left(1-\bar{y}_{2}\right) /(2 \bar{y}(1-\bar{y}))\right)$, we have $0<x_{1}(\alpha)<y_{m}<\bar{y}<x_{2}(\alpha)<1$. 


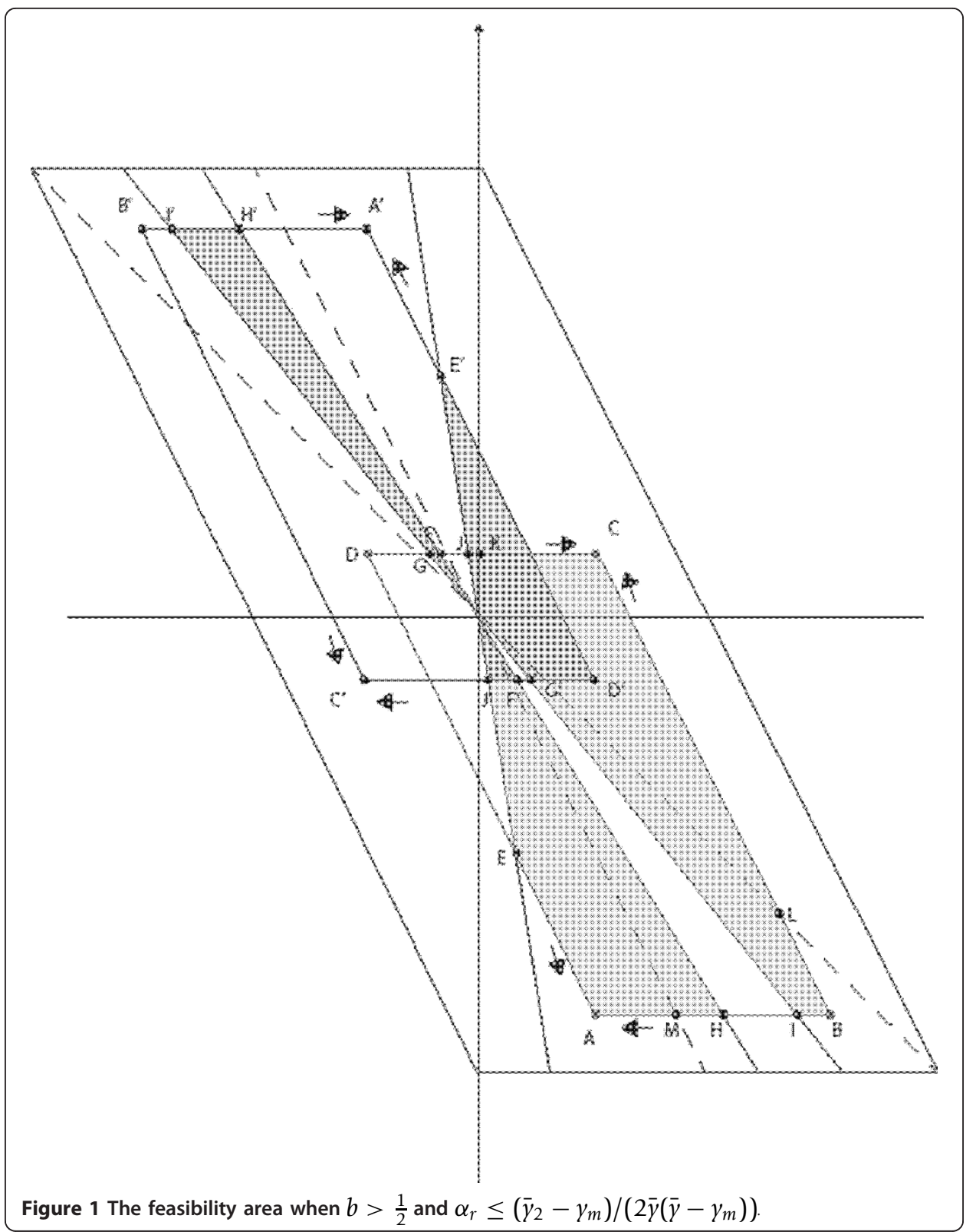

In this case

- if $F\left(x_{2}(\alpha)\right)-F\left(x_{1}(\alpha)\right)<1 / 2$ then $q \in \operatorname{Obj}_{Q T}(t)$ for $u<0$ and $d(q, t)=-u \int_{\left[0, x_{2}(\alpha)\right.} h(x, \alpha) \mathrm{d} F(x)$. For the sake of simplicity we suppose that the open set $\left\{\alpha: F\left(x_{2}(\alpha)\right)-F\left(x_{1}(\alpha)\right)<1 / 2\right\}$ consists of only one open interval (the same type of arguments apply in the general case, when the set is an union of open intervals).

- if $F\left(x_{2}(\alpha)\right)-F\left(x_{1}(\alpha)\right)>1 / 2$ then $q \in \operatorname{Obj}_{Q T}(t)$ for $u>0$ and $d(q, t)=u \int_{\left[x_{1}(\alpha), x_{2}(\alpha)\right]} h(x, \alpha) \mathrm{d} F(x)$. We remark the fact that if $\alpha \in\left(\bar{y}_{2} /\left(2 \bar{y}^{2}\right), 1 /(2 \bar{y})\right]$ then the previous condition is realized.

- if $F\left(x_{2}(\alpha)\right)-F\left(x_{1}(\alpha)\right)=1 / 2$ then there is no objection of $t$. 


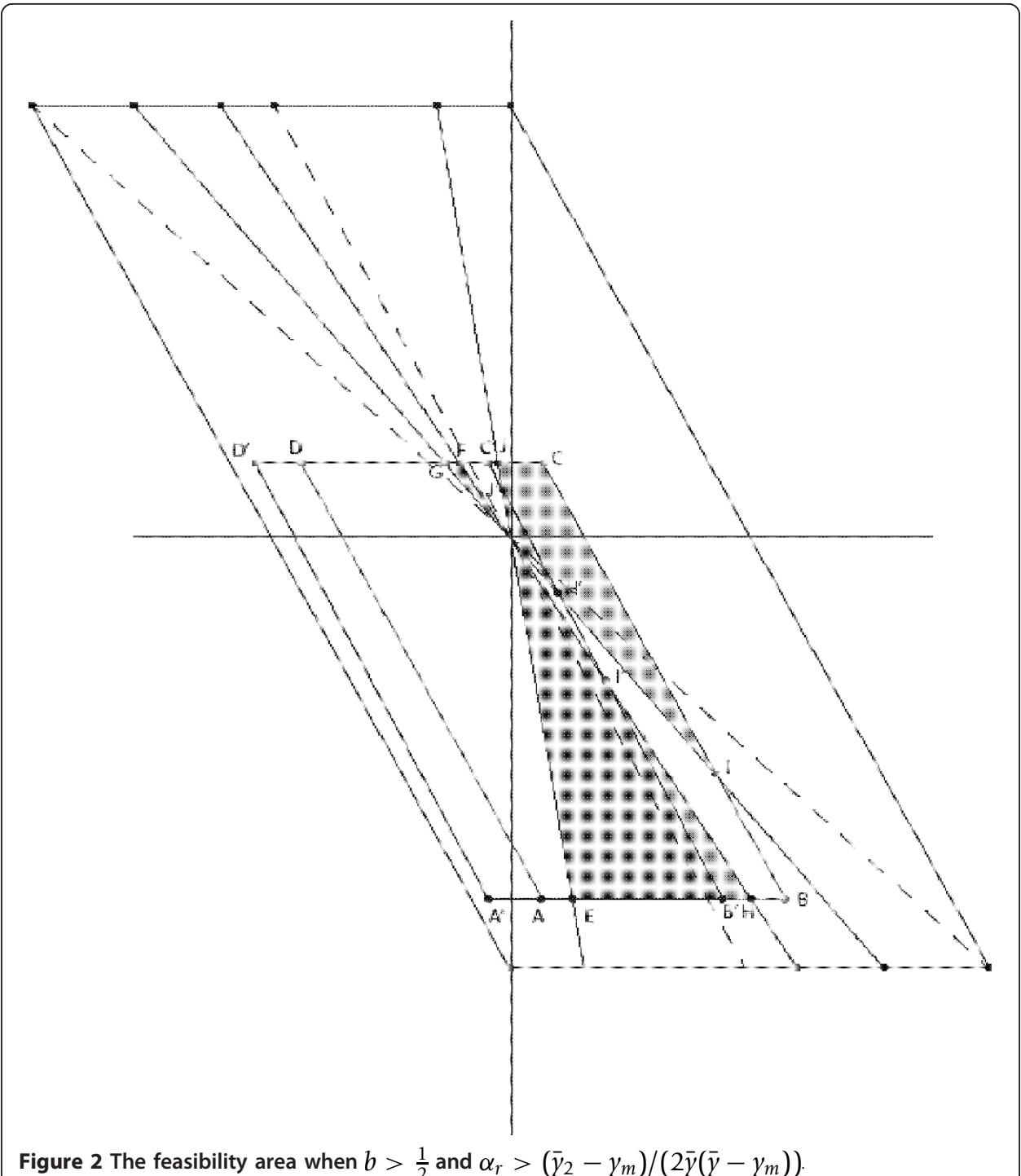

- For $\alpha \in\left(\left(1-\bar{y}_{2}\right) /(2 \bar{\gamma}(1-\bar{\gamma})),\left(\bar{\gamma}_{2}-y_{m}\right) /\left(2 \bar{y}\left(\bar{\gamma}-y_{m}\right)\right)\right)$ since $0<x_{1}(\alpha)<y_{m}<1<x_{2}$ ( $\alpha$ ) we get that $q$ is an objection if $u=\bar{a}-a>0$. In this case, $d(q, t)=u \int_{\left[x_{1}(\alpha), 1\right]} h(x, \alpha) \mathrm{d} F(x)$.

- For $\alpha=\left(\bar{y}_{2}-y_{m}\right) /\left(2 \bar{y}\left(\bar{\gamma}-y_{m}\right)\right)$, since $x_{1}(\alpha)=y_{m}$ and $x_{2}(\alpha)>1$, there is no objection of $t$.

- For $\alpha \in\left(\left(\bar{\gamma}_{2}-y_{m}\right) /\left(2 \bar{y}\left(\bar{y}-y_{m}\right)\right), \infty\right)$ since $y_{m}<x_{1}(\alpha)<\bar{y}<1<x_{2}(\alpha)$, we get that $q$ is an objection if $u<0$. In this case, $d(q, t)=-u \int_{\left[x_{1}(\alpha), 1\right]} h(x, \alpha) \mathrm{d} F(x)$.

Finally, if $u=0$ then $q=(a, \bar{b})$ is an objection of $t$ for each $(a, \bar{b})$ in $\left(F A_{1}\right)$, and $d(q, t)=|v| \int_{[0, \bar{y}]}(\bar{y}-x) \mathrm{d} F(x)$.

For a given $\alpha$ (which represent a direction that passes through the origin), since $d(t$, $q)$ is given by one of the following expressions: $|u| \int_{\left[0, x_{2}(\alpha)\right]} h(x, \alpha) \mathrm{d} F(x)$, $|u| \int_{\left[x_{1}(\alpha), 1\right]} h(x, \alpha) \mathrm{d} F(x), \quad|u| \int_{\left[x_{1}(\alpha), 1\right]} h(x, \alpha) \mathrm{d} F(x) \quad$ or 
$\alpha_{r}>\left(\bar{y}_{2}-y_{m}\right) /\left(2 \bar{y}\left(\bar{\gamma}-y_{m}\right)\right)$ is obtained for the maximum values of $|u|$ or $|v|$. Hence it is sufficient to analyze the behavior of the distance $d(q, t)$ on the border of the feasibility set.

In order to prove the desired inequality, it is sufficient to prove that for each regressive tax function $t_{r}=\left(a_{r}, b_{r}\right), a_{r}<0$, there exists a progressive tax function $t_{p}=\left(a_{p}, b_{p}\right)$ such that $\sup _{q \in \mathrm{Obj}_{\mathrm{Q} T}\left(t_{p}\right)} d\left(q, t_{p}\right)<\sup _{q \in \mathrm{Obj}_{Q T}\left(t_{r}\right)} d\left(q, t_{r}\right)$.

First, we prove the conclusion for $t_{r}=\left(a_{r}, b_{r}\right)$ a regressive tax function for which one of the following two conditions is fulfilled.

$$
\begin{aligned}
& 0 \leq b_{r} \leq 1 / 2 \\
& b_{r}>1 / 2 \text { and } \alpha_{r}=-b_{r} /\left(2 a_{r} \bar{y}\right) \leq\left(\bar{y}_{2}-\gamma_{m}\right) /\left(2 \bar{y}\left(\bar{y}-y_{m}\right)\right)
\end{aligned}
$$

We will present the detailed proof only for the second case (similar arguments apply for the first case). According to the previous discussion, the set $F A_{r}=\left\{\left(u_{r}, v_{r}\right): u_{r}=\bar{a}-a_{r}, v_{r}=\bar{b}-b_{r}, q=(\bar{a}, \bar{b}) \in \operatorname{Obj}_{Q T}\left(t_{r}\right)\right\}$ (see Figure 1) is the union of the interiors polygons $G O F, O E A H$, and $O I B C J$ with the segments $G F, J C, C B$, $B I$, and $H A$.

We shall prove that the progressive tax function $t_{p}=\left(a_{p}, b_{p}\right), a_{p}=-a_{r}, b_{p}=1-b_{r}$ satisfies the desired inequality (actually in both cases, the progressive tax function is the same).

Due to the fact that a part of $F A_{p}=\left\{\left(u_{p}, v_{p}\right): u_{p}=\bar{a}-a_{p}, v_{p}=\bar{b}-b_{p}, q=(\bar{a}, \bar{b}) \in \operatorname{Obj}_{Q T}\left(t_{p}\right)\right\}$ is included in $F A_{r}$, we have to show that the supremum of the distance on the union of the segments $I H^{\prime}$ and $D^{\prime} E^{\prime}$ is smaller than $\sup _{q \in \mathrm{Obj}_{Q_{\mathrm{QT}}}\left(t_{r}\right)} d\left(q, t_{r}\right)$. In fact, it is sufficient to consider only the part of $D^{\prime} E^{\prime}$ for which $v_{p}>0$.

By using the well-known formulas regarding the derivative of parameter depending integrals, we have the following cases (see Figure 1). Also, from now on, we shall use the following notation $h(\alpha)=d\left(q, t_{p}\right)$.

On the segment $E^{\prime} K^{\prime},\left(K^{\prime}\right.$ is the intersection of $E^{\prime} D^{\prime}$ with the ordinate axis), $2 u_{p}+v_{p}=1-\left(2 a_{p}+b_{p}\right), \quad 2 u_{p}+v_{p}=1-\left(2 a_{p}+b_{p}\right) \quad$ and $h^{\prime}(\alpha)=-(1-(2 a+b)) \bar{y} /(1-\alpha \bar{y})^{2} \int_{\left[x_{1}(\alpha), 1\right]}\left(-x^{2}+2 x+\bar{y}_{2}-2\right) \mathrm{d} F(x)$. In the previous equality, the integrand is the most regressive tax function and since $\left.R=\int_{[0,1]}-x^{2}+2 x+\bar{y}_{2}-2\right) \bar{d} F(x)=0$ we obtain that $h^{\prime}(\alpha)<0$. Hence, the values of the distance $d\left(q, t_{p}\right)$ are increasing from $K^{\prime}$ to $E^{\prime}$.

On the part of the segment $K^{\prime} D^{\prime}$ that is situated above the abscissa's axis, similar arguments give us that $h^{\prime}(\alpha)<0$; hence, the values of $d\left(q, t_{p}\right)$ are decreasing from $K^{\prime}$ to $D^{\prime}$.

Combining the previous two results, we see that the supremum of the distance $d(q$, $\left.t_{p}\right)$ on this part of the border is realized at $E^{\prime}$. Due to the symmetry of the Figure 1, the value of $d\left(q, t_{p}\right)$ at $E^{\prime}$ is the same with the value of $d\left(q, t_{r}\right)$ at $E$ (indeed, at $E^{\prime}$ and at $E$ we have the same $\alpha$ and $u_{r}(E)=-u_{p}\left(E^{\prime}\right)$ ).

On the segment I'H', $\alpha \in\left(\bar{\gamma}_{2} /\left(2 \bar{\gamma}^{2}\right),\left(1-\bar{y}_{2}\right) /(2 \bar{\gamma}(1-\bar{\gamma}))\right), v_{p}=b, u_{p}<0$ and $h^{\prime}(\alpha)=b /\left(\bar{\gamma} \alpha^{2}\right) \int_{x_{1}(\alpha), x_{2}(\alpha)}\left(x^{2}-\bar{y}_{2}\right) \mathrm{d} F(x) . \quad$ Since $x_{1}\left(\bar{y}_{2} /\left(2 \bar{y}^{2}\right)=0, x_{2}\left(\left(1-\bar{y}_{2}\right) /(2 \bar{y}(1-\bar{y}))\right)=1\right.$, the integrand in the above derivative is the most progressive tax function (for which $\left.R=\int_{[0,1]}\left(x^{2}-\bar{y}_{2}\right) \mathrm{d} F(x)=0\right)$ we get that $h^{\prime}\left(\left(1-\bar{y}_{2}\right) /(2 \bar{y}(1-\bar{\gamma}))\right)>0, h^{\prime}\left(\left(1-\bar{y}_{2}\right) /(2 \bar{y}(1-\bar{\gamma}))\right)>0$ and the fact that $h^{\prime}$ is an 
increasing function on the considered interval. In consequence the equation $h^{\prime}(\alpha)=0$ has a unique root $\alpha^{*} \in\left(\bar{\gamma}_{2} /\left(2 \bar{y}^{2}\right),\left(1-\bar{y}_{2}\right) /(2 \bar{\gamma}(1-\bar{\gamma}))\right), h^{\prime}$ is negative at the left-hand side of $\alpha^{*}$ and positive at the right-hand side of $\alpha^{*}$.

There are three different cases regarding the position of the point $N^{\prime}$ (the intersection of the line that passes through the origin and whose direction is $\alpha^{*}$ with the segment $\left.A^{\prime} B^{\prime}\right)$ with respect to the segment $I^{\prime} H^{\prime}$ ' at the left-hand side of $I^{\prime}$, in the interior of the segment $I^{\prime} H^{\prime}$, and at the right-hand side of $H^{\prime}$. In all three cases, by using the monotonicity of $h$, we easily obtain that the supremum of the distance $d\left(q, t_{p}\right)$ is the maximum value of $d\left(q, t_{p}\right)$ computed at the points $I^{\prime}$ and $H^{\prime}$. By using again the symmetry of the Figure 1, the values of $d\left(q, t_{p}\right)$ at the points $I^{\prime}$ and $H^{\prime}$ are equal to the values of $d\left(q, t_{r}\right)$ at the points $I$ and $H$. Since $\sup _{q \in \operatorname{Obj}_{Q T}\left(t_{r}\right)} d\left(q, t_{p}\right)$ is attained at one of the points $E, H$, or $I$, and $\sup _{q \in \operatorname{Obj}_{Q T}\left(t_{r}\right)} d\left(q, t_{r}\right)$ is attained at one of the points $A$ or $C$, and the values of the distance at the points $A, C$ are greater than the values of the distance at the points $E, H$, I, we get the desired strict inequality and this part of the proof is complete (see Figure 1).

The remaining part to prove the inequality for $t_{r}=\left(a_{r}, b_{r}\right)$, where $a_{r}<0, b_{r}>1 / 2$, and $\alpha_{r}=-b_{r} /\left(2 a_{r} \bar{y}\right)>\left(\bar{y}_{2}-\gamma_{m}\right) /\left(2 \bar{\gamma}\left(\bar{\gamma}-\gamma_{m}\right)\right)$, is trivial. Similar arguments give us the fact that $\sup _{q \in \mathrm{Obj}_{Q T}\left(t_{r}\right)} d\left(q, t_{r}\right)$ is attained at one of the points $E$ or $C$ (see Figure 2). If the supremum is obtained at the point $C$, by taking $t_{p}=\left(a_{p}, b_{p}\right), b_{p}=b_{r}, a_{p}=\varepsilon$, with $\varepsilon$ sufficiently small, the desired strict inequality follows immediately. If the supremum is obtained at the point $E$, the conclusion follows by taking $t_{p}=\left(a_{p}, b_{p}\right), b_{p}=b_{r}-\varepsilon, a_{p}=$ $3 \varepsilon / 2$, with $\varepsilon$ sufficiently small. This completes the proof.

It should be noted that the above theorem does not have an empty scope. There exists a class of income distribution functions fulfilling all the conditions in the theorem. Take the example of income distribution function in Curt et al. [20]. The exercise to check the condition $F\left(\bar{y}_{2} / \bar{y}\right)-F\left(\left(\bar{y}-\bar{y}_{2}\right) /(1-\bar{\gamma})\right)<1 / 2$ is left to the reader. The rest of the conditions are already checked in that article.

\section{Conclusions}

In the general setup of fixed-income taxation with (absolutely) continuous income distributions, we have mathematically defined the concept of least core and provided a sufficient condition on the policy space such that the former set is not empty. In particular, the least core is not empty for the framework of quadratic taxations, respectively picewise linear tax schedules. Moreover, for fixed-income quadratic taxation environments with no Condorcet winner, we have proved that for sufficiently right-skewed income distribution functions, the least core is characterized by taxes with marginalrate progressivity. Therefore, at least for quadratic taxations, a possible way out from the vote cycling theorem of Hindriks [7] is to consider this less demanding solution concept, but very related to the core.

Note that, even if in the purely redistributive case, voting over tax schemes that satisfy (1)-(4) is not equivalent with voting over income distributions as in Grandmont [8]. Conditions (2) and (3) insure that every tax is increasing with the revenues in such a way that it does not change the post-tax income ranking. Thus, issues like progressivity versus regressivity can be put into discussion once the policy space $U$ is large enough and the least core of the set $U$ is not empty (according to the first theorem in the present paper, this happens for every policy space $U$ that is complete with respect 
to the $L^{1}$ metric). This research opens venues to investigating the stability and progressivity prevalence in income taxation by applying concepts neighboring the core. Therefore, for the future, a more (realistic) case of neither concave nor convex tax functions should be investigated. Moreover, an interesting line of research is toward discrete income distribution functions (see also Moreno-Ternero [22]).

Looking at the implications of the least core in fixed-income taxation environments may provide a real contribution to our understanding of the field; and these implications should be investigated even before considering other less demanding solution concepts or before restricting too much the taxation space.

\section{Endnotes}

${ }^{a}$ Notation: For better comprehensibility of the text, any parameter calculated based on the distribution $F$ is denoted using the letter $y$, e.g., the mean is $\bar{y}$, the median is denoted by $y_{m}$, the non-centered moment of second order is $\bar{y}_{2}$, etc., while $x$ refers to a random income in the interval $[0,1]$. ${ }^{\mathrm{b}}$ When there is no danger of confusion, the explicit dependence on $F$ and $R$ will be dropped.

\section{Acknowledgements}

We thank one anonymous referee for his/her valuable comments. Cristian Litan acknowledges preliminary discussions with Francisco Marhuenda, Luis Corchón, and Oriol Carbonell. Diana Andrada Filip and Paula Curt acknowledge financial support by CNSIS - UEFISCU, project number PNII-IDEI 2366/2008. Cristian Litan acknowledges financial support by CNCSIS-UEFISCSU, project number PN II-RU 415/2010. The usual disclaimer applies.

\section{Author details}

'Department of Statistics, Forecasting and Mathematics, Faculty of Economics and Business Administration, University Babeş Bolyai, 400591 Cluj-Napoca, Romania 'Laboratoire d'Economie d'Orléans, Faculté de Droit, d'Economie et de Gestion, 45067 Orléans, France

\section{Authors' contributions}

The authors jointly worked on deriving the results. All authors read and approved the final manuscript.

\section{Competing interests}

The authors declare that they have no competing interests.

Received: 23 August 2011 Accepted: 16 December 2011 Published: 16 December 2011

References

1. Romer, T: Individual welfare, majority voting, and the properties of a linear income tax. J Public Econ. 4, 163-185 (1975). doi:10.1016/0047-2727(75)90016-X

2. Romer, T: Majority voting on tax parameters. J Public Econ. 7, 127-133 (1977). doi:10.1016/0047-2727(77)90040-8

3. Roberts, K: Voting over income tax schedules. J Public Econ. 8, 329-340 (1977). doi:10.1016/0047-2727(77)90005-6

4. Cukierman, A, Meltzer, H: A political theory of income taxation. In: Meltzer A, Cukierman A, Richards F (eds.) Political Economy. pp. 78-106. Oxford University Press, Oxford (1991)

5. Marhuenda, F, Ortuño-Ortin, I: Popular support for progressive income taxation. Econ Lett. 48, 319-324 (1995). doi:10.1016/0165-1765(95)94399-G

6. Marhuenda, F, Ortuño-Ortin, I: Income taxation, uncertainty and stability. J Public Econ. 67, 285-300 (1998). doi:10.1016/ S0047-2727(97)00054-6

7. Hindriks, J: Is there a political demand for income tax progressivity? Econ Lett. 73, 43-50 (2001). doi:10.1016/S0165-1765 (01)00459-1

8. Grandmont, JM: Fiscally stable distributions under majority voting. Adv Math Econ. 8, 215-230 (2006). doi:10.1007/4431-30899-7_8

9. Carbonell-Nicolau, O, Ok, EA: Voting on income taxation. J Econ Theory. 134, 249-286 (2007). doi:10.1016/j. jet.2006.02.008

10. De Donder, P, Hindriks, J: The politics of progressive income taxation with incentive effects. J Public Econ. 87, 2639-2660 (2003). doi:10.1016/S0047-2727(02)00078-6

11. Roemer, JE: The democratic political economy of progressive taxation. Econometrica. 67, 1-19 (1999). doi:10.1111/14680262.00001

12. Carbonell-Nicolau, O, Klor, EF: Representative democracy and marginal rate progressive income taxation. J Public Econ. 87, 2339-2366 (2003). doi:10.1016/S0047-2727(03)00073-2

13. Einy, E, Holzman, R, Monderer, D: On the least core and the mas-colell bargaining set. Games Econ Behav. 28, 181-188 (1999). doi:10.1006/game.1998.0694 
14. Litan, CM: On the stability of income taxation-a coalitional approach. Virgil Madgearu Rev Econ Stud Res. 1, 68-87http://www.ceeol.com/aspx/issuedetails.aspx?issueid=f87c7825-50dd-4e10-82b7bd04d20c7afb\&articleld=d0599093-a695-42d4-aba3-c158592dfba9 (2010)

15. De Donder, P, Hindriks, J: Majority support for progressive income taxation with corner preferences. Public Choice. 118, 437-449https://springerlink3.metapress.com/content/k60j1rh38730gp12/resource-secured/?target=fulltext. pdf\&sid=4jtt3phlmo2w1sbc23tdxtr5\&sh=www.springerlink.com (2004)

16. Edwards, RE: Measure, Functional Analysis Theory and Applications. Holt, Rinehart and Winston, New York (1965)

17. Ash, RB: Measure, Integration and Functional Analysis. Academic Press, New York and London (1972)

18. Simon, J: Compact sets in the space $L^{P}(0, T ; B)$. Annali di Matematica pura ed applicata. 146, 65-96 (1987)

19. Klor, EF: On the popular support for progressive taxation. J Public Econ Theory. 5, 593-604 (2003). doi:10.1111/14679779.t01-1-00150

20. Curt, P, Litan, CM, Filip, DA: On the majority support for progrssive income taxation when income is fixed and taxation is quadratique purely redistributive. Studia UBB Oeconomica. 56, 43-51 http://studiaoeconomica.ubbcluj.ro/Abstract/ volume561/abstract_03.pdf (2011)

21. Curt, P, Litan, CM, Filip, DA: A mathematical revisit of modelling the majority voting on fixed income quadratic taxations. J Inequal Appl 2010, 1-15http://www.journalofinequalitiesandapplications.com/content/pdf/1029-242X-2010329378.pdf (2010). (Published on-line)

22. Moreno-Ternero, J: Voting over piece-wise linear tax methods. J Math Econ. 47, 29-36 (2011). doi:10.1016/j. jmateco.2010.11.002

doi:10.1186/1029-242X-2011-138

Cite this article as: Curt et al: The least core in fixed-income taxation models: a brief mathematical inspection. Journal of Inequalities and Applications 2011 2011:138.

\section{Submit your manuscript to a SpringerOpen ${ }^{\circ}$} journal and benefit from:

Convenient online submission

- Rigorous peer review

- Immediate publication on acceptance

- Open access: articles freely available online

- High visibility within the field

- Retaining the copyright to your article

Submit your next manuscript at $\boldsymbol{\wedge}$ springeropen.com 